\title{
Práticas Algébricas no Contexto de Projetos Pedagógicos de Modelagem
}

\section{Prácticas Algebraicas en Contexto de Proyectos Pedagógicos de Modelación}

\author{
Fabian Arley Posada-Balvin* \\ ORCID iD 0000-0003-4413-7298 \\ Marcelo de Carvalho Borba** \\ ORCID iD 0000-0003-3101-5486
}

\begin{abstract}
Resumo
Apresentamos uma análise do processo de constituição de práticas algébricas no contexto de projetos pedagógicos de modelagem -PPM-. Partimos da ideia de que as práticas algébricas indicam um conjunto de ações orientadas ao tratamento analítico de quantidades indeterminadas, mas, no contexto dos PPM, se constituem mediante a coordenação de processos de covariação espaço-temporal das quantidades intencionalmente escolhidas, com formas de correlação próprias das estruturas conceituais invariantes que tendem a experimentar um caráter "a-espacial" e "atemporal". Seguindo as ideias da teoria da atividade (ENGESTRÖM, 1999; LEONTIEV, 1978; VYGOTSKY, 1981) e do construto teórico de Seres-Humanos-ComMídias (BORBA; VILLARREAL, 2005) consideramos que tal coordenação se desenvolve como um processo interativo de atores humanos (alunos e professor) e não humanos (artefatos culturais) que entram em atividade de modelagem e imbricam, na singularidade de suas ações, diferentes possibilidades de covariação e correlação. Concluímos que o caráter algébrico das práticas se dá mediante a configuração de uma zona de confluência de significação variacional/particular e correlacional/geral através do uso de diferentes formas de representação simbólicas. O processo é exemplificado mediante um dos momentos desenvolvidos por um grupo de alunos de Biologia da UNESP/Campus Rio Claro, estado de São Paulo, Brasil, quando foram convidados a desenvolver projetos de modelagem em uma disciplina de Matemática no segundo semestre do ano de 2013.
\end{abstract}

Palavras-chave: Projetos Pedagógicos de Modelagem. Mediação Artefatual. Variação. Álgebra escolar.

\section{Resumen}

Presentamos un análisis del proceso de constitución de prácticas algebraicas en el contexto de proyectos pedagógicos de modelación -PPM-. Partimos de la idea que las prácticas algebraicas indican un conjunto de acciones orientadas al tratamiento analítico de cantidades indeterminadas, pero, en el contexto de los PPM, se constituyen mediante una singular coordinación de procesos de covariación espacio-temporal de las cuantidades intencionalmente escogidas, con formas de correlación propias de estructuras conceptuales invariantes que tienden a experimentar un carácter "a-espacial" y "a-temporal". Con base en las ideas de la Teoría de la Actividad (ENGESTRÖM, 1999; LEONTIEV, 1978; VYGOTSKY, 1981) y del constructo teórico SeresHumanos-Con-Instrumentos (BORBA; VILLARREAL, 2005), consideramos que tal coordinación se desarrolla

\footnotetext{
* Doutor em Educação Matemática, Universidade Estadual Paulista (UNESP), Rio Claro, Brasil. Professor do Departamento de Matemática, Universidade Federal do Rio Grande do Norte (UFRN), Natal, Brasil. Endereço para correspondência: Campus Universitário Lagoa Nova (UFRN), Natal, RN, Brasil, CEP: 59078-970. E-mail: fabian@ccet.ufrn.br.

** Doutor em Educação Matemática, Cornell University, EUA. Professor do Departamento de Matemática Universidade Estadual Paulista (UNESP), Rio Claro, São Paulo, Brasil. Endereço para correspondência: Av. 24A, 1515. Bela Vista, Rio Claro, São Paulo, Brasil, CEP: 13506-900. E-mail: mborba @ rc.unesp.br.
} 
como un proceso interactivo de actores humanos (alumnos y profesor) con no humanos (artefactos culturales) que entran en actividad de modelación e imbrican, en la singularidad de sus acciones, diferentes posibilidades de covariación y correlación. Concluimos que el carácter algebraico de las prácticas se da mediante la configuración de una zona de confluencia de significación variacional/particular e correlacional/general a través del uso de diferentes formas de representación simbólica. El proceso es ejemplificado mediante uno de los momentos desarrollados por un grupo de alumnos de Biología de la UNESP/Rio Claro/São Paulo/Brasil, cuando fueron invitados a desarrollar proyectos de modelación en un curso de matemática durante el segundo semestre del 2013.

Palabras clave: Proyectos Pedagógicos de Modelación. Mediación artefactual. Variación. Álgebra escolar.

\section{Introdução}

O ensino formal da álgebra, como atualmente é conhecido, é uma criação do século XIX, mas só na segunda metade do século XX tornou-se uma área de crescente interesse para diferentes pesquisadores em Educação Matemática (PONTE; GUIMARÃES, 2014). Inúmeras pesquisas na área têm mostrado que sua aprendizagem, além de ser interessante como objeto de conhecimento e instrumento que auxilia a compreensão de outras ciências, também é importante por favorecer o desenvolvimento das funções psicológicas superiores (CAI; KNUTH, 2011; STACEY; CHICK; KENDAL, 2004; SUTHERLAND; ROJANO; LINS, 2002; VYGOTSKY, 1986). Parte dessa importância apoia-se na ideia de que a álgebra pode ser vista como um protótipo de operabilidade de formas gerais.

Ao conceber qualquer conceito como expressão de algum tipo de generalidade, e a álgebra como um modelo de tratamento e manipulação de conceitos teóricos, consideramos importante estudar a formação de conceitos algébricos em atividades de produção de conhecimento. Nosso propósito neste trabalho é precisamente esse: discutir alguns aspectos relativos à formação de conceitos algébricos em contextos pedagógicos criados intencionalmente para incentivar a produção de conhecimento em sala de aula.

No entanto, e seguindo as ideias de autores como Kursanov (1966); Prado Jr (1980); Vázquez (2007); Obando, Arboleda e Vasco (2014), entendemos que os conceitos, além de serem cristalizações de ações incorporadas em artefatos culturais, servem de instrumentos para a realização de outras ações e, por isso, formar conceitos algébricos em contextos de produção de conhecimento significa constituir um tipo específico de prática, que denominaremos de prática algébrica.

Como acontece com qualquer prática social, as algébricas são legitimadas 
societalmente ${ }^{1}$ enquanto são desenvolvidas em tempos e espaços determinados com o objetivo de manipular, de alguma maneira, o mundo material em que vivemos. Quando falamos de práticas algébricas, nos referimos a formas de ação orientadas ao tratamento analítico de quantidades indeterminadas que se materializam pelo tratamento de formas gerais mediante o uso de diferentes sistemas de representação simbólica (GODINO et al., 2012; RADFORD, 2010b).

Para atingir nosso objetivo, alunos de uma disciplina de Matemática para biólogos da universidade UNESP/Campus Rio Claro, estado de São Paulo, Brasil, foram convidados a desenvolver Projetos Pedagógicos de Modelagem - PPM - (MALHEIROS, 2004; 2007).

De acordo com Borba (2001; 2011); Borba e Villarreal (2005) isso significa que os alunos foram convidados a escolher uma temática do próprio interesse, a formular pelo menos uma situação-problema relacionada com a temática escolhida, construir argumentos para dar resposta à situação formulada e, finalmente, a produzir um relatório escrito síntese do processo. Tal convite para desenvolver PPM, além de ser uma das tarefas escolares, foi interpretado como um estímulo orientado à produção coletiva de conhecimento em sala de aula.

Duas perguntas tornaram-se de particular importância para nosso propósito: 1) quais ações desenvolvidas no contexto de PPM alcançam o "status" de algébricas? 2) Que tipo de interações pedagógicas favorecem a constituição de práticas algébricas no contexto de PPM? Norteados por estas questões iniciamos este trabalho discutindo o conceito de prática algébrica em contextos escolares, argumentando que quando constituídas como parte do processo de modelagem matemática emergente dos PPM, passam por uma análise variacional. Essa análise implica não só a procura de padrões de variação entre as quantidades que intervêm nas situações-problema formuladas, mas também a construção de correspondências com alguma forma geral de interdependência dessas quantidades covariantes; isto é, algum padrão de correlação.

Na sequência, discutimos algumas ideias metodológicas. Para isso, consideramos que a constituição de prática algébricas em contextos de PPM deve ser compreendido como um fenômeno social que implica a criação de condições para transitar de sentidos e significados variacionais para outros de caráter correlacional.

Adotamos assim, uma perspectiva metodológica favorável a processos

\footnotetext{
${ }^{1} \mathrm{O}$ caráter societal das práticas indica que o processo de legitimação é feito por certas instituições de acordo com o contexto particular em que são desenvolvidas.
} 
intervencionistas, em que diferentes atores entram em atividade e interagem mediante o uso intencionado de diversos artefatos culturais materiais e simbólicos, em nosso caso particular, carregados principalmente de significação matemática. Com a intenção de exemplificar algumas das caraterísticas do processo, finalizamos nossa discussão apresentando um episódio em que um grupo de alunas interagem com o professor e dois monitores da disciplina, motivados pela análise de um problema emergente do PPM por elas desenvolvido.

\section{As práticas algébricas e o ensino da álgebra}

Uma das preocupações que tem movimentado o ensino da álgebra está relacionada com a necessidade de determinar o momento mais adequado para começar esse processo. Algumas discussões convergiram em considerar que, pelo grau de abstração e generalidade da álgebra, o mais recomendável era que os alunos entrassem em contato com essa área depois de ter tido pelo menos cinco ou seis anos de experiência com a Matemática escolar, principalmente com a aritmética (LINS; KAPUT, 2004; PONTE; GUIMARÃES, 2014). Os principais argumentos dessa conclusão descansam nos conteúdos curriculares que, supostamente, "deviam ser ensinados" de acordo com certa faixa etária.

Com o ingresso e popularização das tecnologias computacionais nos contextos escolares, essa discussão foi além de questões relacionadas aos conteúdos (BEDNARZ; KIERAN; LEE, 1996). Dada a capacidade que este tipo de tecnologias tem para processar grandes quantidades de informação e de executar ações e operações consideradas exclusivamente dos seres humanos, podendo, não só transformar expressões simbólicas e resolver diferentes tipos de equações, mas também operar conjuntamente com outros sistemas simbólicos matemáticos tais como gráficos, tabelas e simulações, outros assuntos de cunho epistemológico e metodológico passaram a ser de interesse geral. É o caso de discussões relacionadas ao tipo de situações-problema solucionáveis com técnicas algébricas e às possibilidades que oferecem aos contextos escolares para desenvolver processos de ensino da álgebra.

A análise e tratamento sistemático de padrões de variação, o estudo de processos de generalização e a construção de modelos matemáticos em situações das ciências naturais e sociais passaram a serem assumidas como contextos importantes para a formação de conceitos algébricos (BEDNARZ; KIERAN; LEE, 1996; BLANTON; KAPUT, 2011; KIERAN, 2004). Logo, as fronteiras que delimitavam os conteúdos e os tipos de problemas algébricos que deveriam e poderiam ser tratados na escola tornaram-se cada vez mais 
confusos (GODINO et al., 2012).

Dessa discussão desprendem-se dois aspectos imbricados e em permanente tensão, cuja co-determinação deve ser levada em conta quando se trata de desenvolver processos de formação de conhecimento algébrico na escola: (1) entender que atualmente a álgebra constitui um campo teórico próprio da Matemática e (2) reconhecer que pode ser entendida como uma particular forma de pensar. O primeiro aspecto refere-se à natureza organizativa dos objetos e conceitos algébricos, mas também de como as afirmações e resultados respondem a critérios de veracidade internos à Matemática. Já o segundo aspecto refere-se a atos reflexivos e operações mentais e corporais que os sujeitos desenvolvem em tempos e espaços determinados com o objetivo de manipular, transformar e controlar o mundo material em que vivemos e, desse modo, satisfazer as necessidades de existência e transcendência.

Entender a álgebra escolar nessas condições permite considerá-la como uma particular prática matemática sempre em desenvolvimento que depende de processos de legitimação societal e requer espaços pedagógicos intencionados para sua constituição, começando nos primeiros anos de escolaridade (BLANTON; KAPUT, 2011; LINS; GIMENEZ, 2001; POSADA et al., 2006; RIBEIRO; CURY, 2015). Do mesmo modo, quando os mecanismos pedagógicos usados para a formação de conceitos algébricos são situações-problema das ciências naturais e sociais, envolve, pelo geral, o estudo sistemático de qualidades e quantidades que variam em campos particulares de variação. Em outras palavras, diremos que requer uma permanente ativação, o que autores como Posada et al. (2006), Cantoral e Farfán (1998) chamam de pensamento variacional e Blanton e Kaput (2011) de perspectiva funcional.

\section{As práticas algébricas em contextos de modelagem: o papel da variação}

Quando afirmamos que na álgebra confluem pelo menos dois aspectos intimamente conectados: ser um ramo da Matemática e indicar uma maneira particular de pensar (pensamento algébrico), estamos querendo dizer que ela se constitui como parte de um processo cultural e, como tal, indica uma forma de comportamento prático legitimado societalmente. Nesse sentido, a álgebra deve ser entendida não só como produto de alguma prática social, mas também como instrumento para o desenvolvimento de outras práticas culturalmente diferenciadas. Em outras palavras, ao realizar ações orientadas intencionalmente a transformar o mundo material para satisfazer as necessidades usando (ou produzindo) conceitos, objetos e relações legitimadas com o significado de algébricas, 
podemos dizer que estamos numa prática algébrica.

Como o termo "algébrica" está adjetivando a "prática", indica que é uma particular forma intencionada dos sujeitos organizarem suas ações e reflexões, da mesma forma como seria uma "prática geométrica", uma "prática política", uma "prática científica" etc. Mas, ao mesmo tempo, indicaria que é possível diferenciá-la desses outros tipos de práticas. Cada uma delas designa formas diferentes de manipular e legitimar as ações que os sujeitos desenvolvem com o propósito de transformar intencionalmente o mundo em que vivem.

Mas, quais as caraterísticas de uma prática dita "algébrica"? Diversos autores (BLANTON; KAPUT, 2011; CHARBONNEAU, 1996; GODINO et al., 2012; KIERAN, 2004, 2006; LINS, 1992; RADFORD, 2010a; RIBEIRO; CURY, 2015) reconhecem ser uma pergunta de difícil resposta, não só porque depende do contexto histórico-cultural a que se faça referência, mas também porque implica uma tentativa de clarificação das difusas fronteiras a respeito de outras formas de práticas matemáticas (aritméticas e geométricas, por exemplo).

No entanto, mesmo com a falta de consenso de delimitação e caraterização mediante uma única definição, é possível mencionar dois aspectos de particular interesse associados às práticas algébricas. Em primeiro lugar, nesse tipo de prática são tratadas relações entre quantidades de natureza indeterminadas operadas fundamentalmente de forma analítica ${ }^{2}$. Isso significa que numa prática algébrica opera-se com quantidades e relações desconhecidas (variáveis, incógnitas, parâmetros, números generalizados) como se fossem aritmeticamente conhecidas e cuja operabilidade torna-se possível pela mediação de distintos sistemas simbólicos formais $^{3}$ (especialmente alfa numéricas, mas não unicamente).

E, em segundo lugar, mesmo os processos de generalização empírica e os de tratamento de quantidades que variam conjuntamente (covariação) não serem assumidos como processos tipicamente próprios da álgebra, são considerados aspectos centrais para o desenvolvimento de práticas algébricas. Isso sugere que, embora tais processos não indiquem necessariamente um tratamento analítico de quantidades indeterminadas gerais ${ }^{4}$, podem ser

\footnotetext{
${ }^{2}$ De acordo com Klein (1992, nota 232) a análise, no pensamento algébrico, pode ser entendida com um duplo sentido: de um lado, como um método para achar verdades matemáticas, e, de outro, para caracterizar que o desconhecido pode ser tratado com o mesmo "status" de aquilo que é conhecido. Ver também Viète (1983).

${ }^{3}$ Godino et al. (2012) e Radford, (2010a) argumentam que o uso de letras é só uma possibilidade com grandes potencialidades operacionais e cognitivas, mas outras formas simbólicas, frutos de processos históricos e culturais particulares, poderiam mediar as formas do pensar algébrico.

${ }^{4}$ Entre o "genérico" e o "generalizado" há diferenças substanciais. Uma situação "generalizada" emerge quando se passa a falar do que é comum a um conjunto de casos particulares. Ao passo que a situação "genérica" emerge quando tratamos diretamente daquilo que é geral numa situação, sem a intermediação dos casos particulares
} 
vistos como contextos potencialmente pertinente para a constituição de diversos graus de abstração e generalização algébrica (KIERAN, 2004; MUNZÓN; BOSCH; GASCÓN, 2015).

Quando situações provenientes das ciências naturais e/ou sociais precisam ser estudadas, a construção de padrões de variação das quantidades que intervêm na situação torna-se um aspecto necessário, em que, os símbolos usados nas expressões impregnam-se de significados dinâmicos, permitindo o estudo dos campos de variações de certas quantidades, quando outras se fazem variar intencionalmente; isto é, uma análise covariacional. Um segundo aspecto importante é a constituição de formas gerais de interdependência dessas quantidades covariantes indicando uma análise correlacional. Desse modo, é possível afirmar que no momento em que um sujeito precisa realizar alguma prática algébrica sobre alguma situação das ciências naturais, sociais ou da vida cotidiana (produzir modelos algébricos), passa constantemente de uma abordagem variacional para outra correlacional e vice-versa.

De acordo com Posada e Villa (2006), a coordenar quantidades covariantes com formas de correlação associadas, não é um processo simples. Mesmo alguém raciocinando variacionalmente ajustado à situação de interesse, nem sempre consegue determinar correlações entre as quantidades pertinentes da situação; ou seja, determinar algum padrão invariante de correlação entre as variáveis. E isso, entre outras coisas, porque nem sempre é possível $^{5}$.

\section{Metodologia para a análise}

Da variedade de perspectivas sobre modelagem que na atualidade permeiam o campo da Educação (ARAÚJO, 2010; KAISER; SRIRAMAN, 2006), assumimos a proposta por Borba (2011; 2001); Borba e Villarreal (2005), que incluem, como um de seus princípios pedagógicos, incentivar a produção de conhecimento em sala de aula como um meio para a formação de pensamento crítico. Nessa proposta, os alunos são convidados a formular e resolver situações-problema no contexto de temas escolhidos de acordo com seus próprios interesses. A principal intenção é criar um espaço pedagógico favorável à execução de ações análogas às realizadas por pesquisadores quando desenvolvem suas investigações: formular, delimitar e resolver situações-problema. Autores como Malheiros (2004; 2007) têm denominado essa proposta de projetos pedagógicos de modelagem - PPM.

(LINS, 1992; LINS; GIMENEZ, 2001, p. 114).

${ }^{5}$ Em geral as variações de natureza probabilística são casos representativos de esse tipo de situações. 
Dado que as ações desenvolvidas nos PPM são intencionalmente orientadas à produção coletiva de conhecimento em contextos de sala de aula, o intuito é que a tendência seja produzir não só conhecimento empírico, mas também sistemático, organizado e conceitual; isto é, um processo de interpelação constante entre conhecimento teórico e espontâneo (VYGOTSKY, 1986). Um caso particular é quando, pelas caraterísticas de cada projeto, faz-se necessária a produção de processos de quantificações e/ou de tratamento de formas espaciais criando um espaço potencial para a constituição de práticas algébricas.

Para estudar o processo de constituição das possíveis práticas algébricas no contexto dos PPM, focamos a atenção nas caraterísticas das ações e interações entre atores humanos e não humanos, pois de acordo com Borba (2001) e Borba e Villarreal (2005), nos processos de produção de conhecimento, tais interações devem ser compreendidas como unidades básicas de análise. Para nosso caso, os atores humanos foram alunos, professor e monitores que participaram, no segundo semestre de 2013, de uma disciplina de Matemática Aplicada para o curso de Biologia da Universidade Paulista "Júlio Mesquita Filho" (UNESP), campus de Rio Claro, São Paulo/Brasil, e os não humanos, o conjunto de artefatos culturais materiais e simbólicos.

O professor solicitou aos alunos que em grupos de, no mínimo, quatro e, no máximo, seis integrantes escolhessem um tema de interesse coletivo, formulassem pelo menos uma situação-problema e/ou pergunta de pesquisa referente ao tema escolhido, e construíssem argumentos orientados a dar possíveis soluções ao problema e/ou responder à pergunta formulada. O produto final era um texto escrito, síntese do processo de investigação e uma apresentação oral para o resto da turma. Os alunos foram acompanhados ativamente pelo professor e um dos monitores da disciplina ${ }^{6}$ durante o semestre todo, produzindo um conjunto de registros escritos (diferentes versões de relatório de pesquisa, os "slides" da apresentação oral); audiovisuais (gravações em vídeo ou só em áudio de diversas discussões acadêmicas dentro e fora de sala de aula); e, entrevistas livres e semiestruturadas. A organização da informação baseou-se nos princípios da teoria da atividade (LEONTIEV, 1978; ENGESTRÖM, 1999) e no método de estimulação dual sugerido por Vygotsky (1981) ${ }^{7}$.

Neste caso, o convite do professor foi interpretado como o primeiro estímulo orientado a detonar a necessidade de entrar na atividade de produção de conhecimento. A partir desse

\footnotetext{
${ }^{6} \mathrm{O}$ professor e um dos monitores são os autores deste artigo.

${ }^{7} \mathrm{O}$ método consiste em apresentar aos alunos dois tipos de estímulos em sequência: o primeiro, um problema que estimula a realização de ações e o segundo que oferece a possibilidade de construir os meios para solucioná-lo (DANIELS, 2003).
} 
momento, um conjunto de novos estímulos foram propostos, à medida que os alunos envolvidos na atividade de modelagem assim o precisassem. Ambos tipos de estímulos foram entendidos como conjunto de atos instrutivos orientados a instigar processos coletivos de produção de conhecimento mediante a interação entre os sujeitos com diferentes níveis de experiência relacionadas com a temática escolhida (mediação pedagógica intersubjetiva), auxiliados por artefatos culturais (mediação artefatual).

As análises partiram da ideia de que as práticas sociais, em particular as algébricas, se constituem em longos períodos de tempo mediante a troca de sentidos, significados, perspectivas e intencionalidades que vão se incorporando nos sistemas de artefatos culturais com que são realizadas as ações, interações e processos de comunicação intra e intersubjetiva.

Desse modo, optamos por uma metodologia baseada nos princípios da microetnografia (STREECK; MEHUS, 2005) e de aproximação semiótica favorável ao estudo das mudanças no comportamento dos sujeitos que entram em atividades específicas (RADFORD; SABENA, 2015). Focamos a atenção principalmente em três aspectos: 1- processos intencionados de envolvimento dos sujeitos; 2- evolução no tempo da moldagem recíproca dos diferentes sentidos e significados de acordo com as caraterísticas e experiências de cada sujeito e das potencialidades dos artefatos utilizados; e 3- a busca de sintonia das ações próprias da atividade modelagem e as correspondentes ações das práticas potencialmente legitimadas como algébricas.

Embora o conjunto de dados tenha sido coletado durante um semestre acadêmico, por questão de espaço não é possível descrever detalhadamente todo o processo ${ }^{8}$. Em vez disso, para ilustrar algumas das análises realizadas, na continuação apresentaremos um episódio em que, durante a apresentação oral do projeto, o professor e os monitores da disciplina interagem com um grupo de alunas motivado pelo estudo de um problema proposto no contexto do PPM desenvolvido. Apresentamos as ideias em dois momentos. No primeiro, discutimos o papel do conceito de covariação na singularidade da relação entre as quantidades pertinentes da situação. No segundo, centramos a atenção no conceito de correlação correspondente como ponto de ancoragem para o tratamento de formas de generalidade, isto é, ações orientadas a constituir práticas algébricas.

\section{Localização de cetáceos em mar aberto: um exemplo}

\footnotetext{
${ }^{8}$ Convidamos ao leitor para ler o relatório completo de pesquisa que se encontra no acervo da biblioteca da UNESP/Campus Rio Claro/SP/Brasil
} 
Um dos grupos, formado por cinco alunas ${ }^{9}$, desenvolveu o projeto intitulado “Cetáceos: um enfoque acústico", cujo objetivo voltou-se para o estudo das caraterísticas dos sons emitidos por alguns cetáceos e a forma como os utilizam para se alimentar, se comunicar, se reproduzir e se localizar no espaço marítimo. O projeto foi dividido em três partes. $\mathrm{Na}$ primeira, apresentaram um conjunto de conceitos relacionados com a Física do som. $\mathrm{Na}$ segunda, mostraram algumas aplicações desses conceitos para os sons emitidos por baleias e golfinhos, explicando como, de acordo com as características encontradas, é possível classificá-los. Na terceira parte, foi explicada uma das técnicas usada por cientistas para localizar cetáceos em mar aberto.

O episódio que vamos apresentar emerge da terceira parte: a partir de um problema que, de acordo com as alunas, foi tomado da "Internet" com o fim de mostrar uma aplicação da matemática na resolução de um problema relacionado à localização de um cetáceo em mar aberto a partir da captura dos sons por ele emitido. A técnica explicada pelas alunas envolvia o uso de vários hidrofones arranjados em diferentes formas. De acordo com elas, os hidrofones recebem os sons emitidos pelo animal, que depois é processado por um computador localizado em um barco relativamente próximo. A tese central era que, calculando as diferenças entre os tempos que cada hidrofone leva para capturar o som é possível determinar o ponto de localização do cetáceo com margens confiáveis de erro, levando em conta que a velocidade do som na água tem um valor constante de aproximadamente $1500 \mathrm{~m} / \mathrm{s}$.

\subsection{Primeiro momento: estudando a singularidade da relação particular-geral}

Para exemplificar o processo, as alunas apresentaram uma situação que envolvia três hidrofones arranjados linearmente. A situação discutida foi: considerando que um pesquisador captura o som emitido por uma baleia com uma diferença de tempo de 0,006 segundos entre o primeiro e o segundo hidrofone e de 0,0007 entre o primeiro e o terceiro hidrofone, determinar as coordenadas cartesianas onde a baleia deve estar localizada. Uma das alunas explicou que os alicerces da técnica apresentada se achavam no conceito matemático de hipérbole $e^{10}$.

\footnotetext{
${ }^{9}$ Os participantes da pesquisa autorizaram o uso de seus nomes próprios. Raphaela, Juliana, Gabriella, Nahanna e Virginia eram alunas de primeiro ano de Biologia; Marcelo o professor; Carlos e Lucas, monitor e estagiário da disciplina, respectivamente; e Fabian, além de monitor, tinha o papel de pesquisador principal.

${ }^{10}$ As ideias aqui apresentadas são uma transcrição textual do vídeo da apresentação oral das alunas e as imagens foram tomadas da gravação e/ou do trabalho escrito. De acordo com elas, a situação foi tomada da internet e a apresentaram mesmo manifestando falta de compreensão do problema. Embora há alguns erros conceituais e
} 


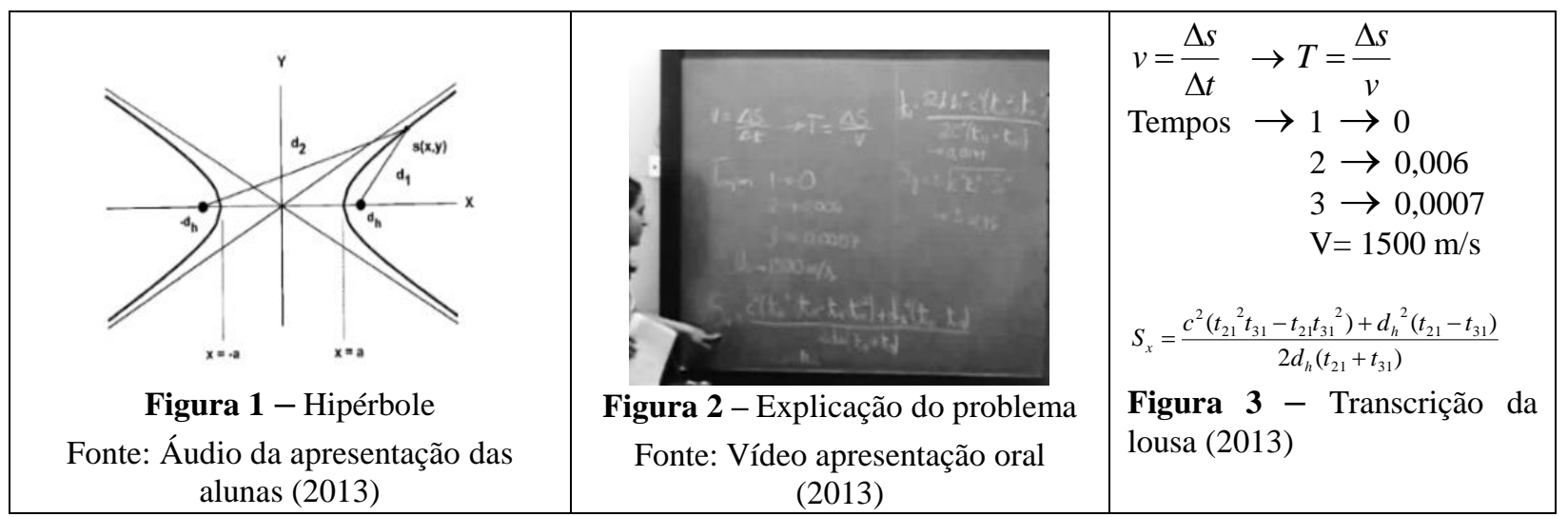

Raphaela: os hidrofones foram colocados a uma distância d1, d2 e d3. Eu coloquei aqui [apontando para o quadro] as equações (figura 2 e 3). A gente sabe da física que a velocidade é a distância pelo tempo. E vamos supor que o tempo que demora o som para chegar até o hidrofone um é zero segundos ${ }^{11}$, até o hidrofone dois 0,006 segundos e até o hidrofone três 0,0007 segundos. A velocidade do som na água é $1500 \mathrm{~m} / \mathrm{s}$. Aqui [mostrando o gráfico que aparece na tela do computador] é uma hipérbole (figura 1). Esta parte da hipérbole [mostrando a asa direita da hipérbole] representa o som que está sendo transmitido pelo animal e aqui [mostrando a asa esquerda da hipérbole] o é o som que está voltando. Aqui já está pronta a fórmula da hipérbole (figura 2 e 3) e posso ir substituindo os valores que eu tenho: $t_{21}$, o tempo que demora entre o hidrofone 1 e 2; e, t3l, entre o hidrofone 1 e 3. A equação $S_{x}$ é para os valores $x$ do ponto cartesiano onde a baleia está.

(Áudio da apresentação oral da aluna, 2013).

Uma vez culminada a apresentação, o professor preocupado por algumas imprecisões conceituais da aluna, interveio indagando-as pontualmente sobre a relação do fenômeno físico e o conceito matemático de hipérbole que, segundo elas, oferecia a solução ao problema formulado.

Marcelo: Por que o conceito matemático hipérbole é o que, segundo vocês, descreve e resolve o problema? Por que não uma parábola, por exemplo?

(Áudio da intervenção do professor após apresentação oral da aluna, 2013).

Todas as alunas ficaram caladas por um tempo com gestos de não saber explicar por que precisamente a Matemática desse lugar geométrico permite desenvolver a técnica para resolver o problema apresentado. No entanto, Raphaela (talvez por se sentir responsável por essa parte do trabalho) atreveu-se a dar uma resposta, repetindo o que já tinha explicado na apresentação, mas neste momento acrescentado alguns gestos com os braços para enfatizar o já dito:

Raphaela: $O$ som da baleia que faz assim [mostrando com os braços a forma de um dos

ideias confusas, não é de nosso interesse discutir e/ou corrigir aqui esses erros conceituais, pelo contrário, alguns deles ajudaram nas análises cognitivas e na melhor compreensão do processo de constituição de práticas algébricas.

${ }^{11}$ Esta afirmação indicaria que a baleia está no mesmo lugar do hidrofone 1. 
ramos da curva] e depois quando volta e faz assim [mostrando com os braços a forma do outro ramo da curva]. Então o gráfico fica, um que vai porque ela emitiu o som, e o outro que volta porque o som voltou [mostrando de novo com os braços a forma das duas asas da curva]. Fica assim a imagem de uma hipérbole como mostrado na figura.

(Áudio da resposta da aluna à pergunta do professor, 2013).



Figura 4 - Aluna explicando o movimento da onda sonora Fonte: Vídeo apresentação oral (2013)

A partir da explicação verbal, acompanhada dos gestos realizados (Figura 4), confirma-se que, de acordo com campo de significação da aluna, tanto da situação física quanto do conceito matemático, além de estar reconhecendo e discriminando algumas quantidades pertinentes da situação, também está determinando formas de covariação entre elas. Com essa informação, realiza uma primeira tentativa de coordenação com uma das possíveis formas de correlação, utilizando a representação do gráfico da hipérbole. Neste sentido, o gráfico representa outra maneira de singularizar a particular relação de covariação entre as quantidades identificadas. No entanto, percebe-se na explicação, que a aluna está fazendo uma associação por analogia da forma geométrica do gráfico com a trajetória da onda sonora, o que indica que o gráfico não está sendo necessariamente usado como uma representação significante (DUVAL, 1999), da forma geral (correlação) de interdependência das quantidades covariantes.

A falta de compreensão da correspondência entre a covariação e a correlação é evidenciada em pelo menos dois aspectos. Em primeiro lugar, porque, caso fosse como a aluna explicou, estaria se referindo a uma onda sonora que realmente tem frente circular (esférica) e não hiperbólica (MEDWIN; CLAY, 1998). Em segundo lugar, porque, de acordo com a explicação e o esquema mímico mostrado pela aluna, interpreta-se que a forma gráfica da hipérbole indicaria que a onda sonora emitida pelo animal viaja em direção aos hidrofones (primeiro ramo da hipérbole), bate contra eles e volta (segundo ramo da hipérbole). Nesse caso, o gráfico não poderia ser a melhor representação dessa situação, pois o reflexo da onda que volta interferiria na onda emitida e, na hipérbole, os ramos do gráfico não se cortam.

O professor é consciente que a aluna não está conseguindo coordenar adequadamente os padrões de covariação das duas quantidades físicas particulares: tempo e distância que 
definem o movimento do som emitido pela baleia, com a correlação entre essas mesmas quantidades encapsuladas nos sistemas simbólicos matemáticos - gráfico e simbólico-analítica (a fórmula) - que representam o conceito de hipérbole. Podemos interpretar que as alunas não estão conseguindo modelar matematicamente o fenômeno físico, pois o conceito matemático não está sendo usado como padrão geral de correlação entre as duas variáveis (modelo matemático). Isso talvez explique porque a fórmula, mesmo sendo escrita no quadro, não foi usada na explicação e na resposta dada à pergunta do professor.

$\mathrm{O}$ professor fez dessa situação emergente um espaço pedagógico adequado para discutir com as alunas algumas ideias sobre o processo da modelagem matemática. Especificamente, aproveitou o momento para criar condições pedagógicas em que fosse possível fazer aparecer na consciência das alunas (aprendizagem) o conceito matemático hipérbole (padrão geral) e seu potencial para auxiliar a compreensão e solução da situaçãoproblema formulada (um caso particular). Interpretando Kursanov (1966); Davidov (1988), o propósito era fazer compreender a hipérbole não só como conceito matemático, mas também seu caráter de modelo e o potencial papel na compreensão, controle e transformação da situação que estava sendo estudada. Por isso, pediu voltarem ao gráfico e comentou:

Marcelo: Após a explicação de Raphaela eu ainda não consegui entender bem porque a hipérbole aqui, mas talvez entre eu, Lucas e Fabian podemos ajudar vocês entender melhor esse conceito matemático e entre todos estudamos como resolver o problema. Para isso, primeiro vamos tentar decifrar a informação que aparece aqui no gráfico e depois iremos à fórmula. $O$ que é aquele dl e d2? [mostrando o gráfico da hipérbole apresentada na Figura 1]

Raphaela: É a distância da baleia a cada microfone ${ }^{12}$.

Marcelo: $O$ que significa o ponto $s(x, y)$ ?

Raphaela: $O$ ponto s(x,y) mostra onde é que a baleia está.

Marcelo: E o que é dhe -dh?

Raphaela: São os microfones

Marcelo: Agora alguma de vocês saberia me dizer porque o gráfico representa uma hipérbole?

(Âudio do diálogo entre professor e alunas, 2013).

Após um tempo de silêncio, gestos corporais, olhares e sons indicadores de não saber responder, Fabian toma a palavra e disse:

Fabian: Podemos lembrar que se a diferença entre $d_{1}$ e $d_{2}$ é uma constante, então se pode explicar por que representa uma hipérbole.

(Vídeo apresentação oral, 2013).

Seguidamente, o professor toma de novo a palavra:

Marcelo: O que Fabian disse é a caraterística principal da hipérbole. Agora o problema é encontrar porque o problema se resolve com esse conceito. Onde está essa diferença

\footnotetext{
${ }^{12}$ A aluna também foi indagada pelo que tinha acontecido com o terceiro hidrofones, mas não soube responder.
} 
constante no fenômeno? Pensemos em explicar primeiro qualitativamente o que é que leva a assumir o conceito de hipérbole, para depois usar as fórmulas.

Raphaela: É um problema muito complexo e reconhecemos que há muitas coisas que não entendemos. Por exemplo, não entendemos a equação e todas as passagens para resolvê-la. Marcelo: Não, mas primeiro precisamos saber dar uma explicação qualitativa do porquê dessa situação implicar em usar precisamente a hipérbole. Depois podemos entender as passagens da equação.

(Áudio do diálogo entre professor e alunas, 2013).

Destes diálogos, queremos ressaltar um elemento de particular importância relacionado à constituição das práticas algébricas em contextos de PPM. Quando se usa a modelagem como proposta pedagógica para o ensino da Matemática, as respostas matematicamente exatas, certas e acabadas deixam de ser uma prioridade para dar passo ao eventual, à incerteza, ao "erro" e, principalmente, à desconstrução de configurações categóricas de ser e não ser. Percebe-se, por exemplo, como professor e alunos vão se tornado partícipes da mesma atividade, sem que nenhum deles seja assumido como único portador do conhecimento, nem provedor de respostas certas, mas atores com a potencialidade de contribuir, a partir das próprias experiências, com ideias para tentar compreender e resolver a situação-problema.

Para todos eles, o conceito de hipérbole tinha sido tema de estudo em momentos anteriores, e, embora cada ator tivesse experiências diversas com esse conceito, estavam ante algo "novo", não porque era desconhecido, mas porque conceitos matemáticos "velhos" podem tornar sua presença em novidade, dependendo da situação que está sendo tratada. Visto assim, a adjetivação da prática como algébrica não é feita unicamente por legitimação externa, mas também pela atualização dos sentidos e significado que são produzidos na singularidade da situação. A prática não é algébrica em si, mas torna-se algébrica pela troca de experiências, interpretações, perspectivas e intencionalidades dos diferentes atores, que vão entrando em sintonia mediante as diferentes interações intersubjetivas e pela troca de artefatos culturais carregados de diferentes significações, dentre elas de significação algébrica.

Para o caso que estamos tratando, destacamos especialmente o papel da linguagem comum e das linguagens conceituais da Matemática e das outras áreas como da Física e da Biologia. No entanto, não se trata de assumir o processo como uma tradução direta do campo de significação variacional da situação física para o campo de significação correlacional matemática e vice-versa, mediada pelas palavras. Essa interação é de natureza bem mais complexa, pois, dentro dos diferentes campos de significação, os sujeitos agem de maneiras diferentes sobre a relevância dos fatos, das justificativas, das afirmações, da aceitação (ou não) das declarações, das crenças, dos interesses, das intencionalidades e das regras de 
validação dos raciocínios. Desse modo, a constituição de práticas algébricas em contextos de PPM, deve ser entendida como um processo que muda com o tempo de acordo com as condições culturais sob mecanismos de legitimidade institucional.

Tanto na apresentação da aluna, quanto no diálogo seguinte com o professor, percebese como cada um deles manifesta as intencionalidades respondendo aos significados da particular institucionalidade que representam. Raphaela, por exemplo, explicou suas compreensões mediante um conjunto de palavras, fórmulas, figuras e formas gráficas, ilustrando sua compreensão sobre a covariação das variáveis físicas e a respectiva correlação matemática com o conceito de hipérbole. Mas também um conjunto de gesticulações e tons de voz que combinados com as outras formas simbólicas tinham o propósito de explicar o processo, cumprindo assim com a necessidade de responder à tarefa escolar que lhes foi proposta e que, vale à pena mencionar, representava uma "nota" avaliativa na disciplina.

Por outro lado, para o professor, que se encontrava num campo de significação diferente do das alunas e cujo viés era mais próximo da institucionalidade científica, a explicação delas não foi totalmente bem-sucedida. Por isso, as incentivou para que revisassem a coerência dos argumentos científicos apresentados, mediante um conjunto de ações identificados como atos instrutivos orientados a estimular a produção de significados teóricos que respondessem correlacionalmente ao fenômeno empírico de variação entre as quantidades. É o caso da orientação dada, de realizar primeiramente uma análise qualitativa e, posteriormente, pensar na análise correlacional; isto é, primeiro fazer uma análise variacional e depois as "passagens" das equações com as que explicariam a correlação do fenômeno fisco. Ou seja, o significado matemático algébrico do conceito hipérbole in situ (espaço-temporal).

\subsection{Segundo momento: tomando consciência do papel da correlação}

Os propósitos dos atos instrutivos, por estarem carregados de padronizações culturais, além de provocarem aprendizagem, servem como ancoragem para produzir desenvolvimento nos sujeitos e limitar ou impulsionar novos comportamentos. O padrão invariante "diferença constante" codificado no conceito de hipérbole é um exemplo de padronização cultural constituído historicamente. Dado que a experiência teórica das alunas com esses conceitos era menor que a do professor, ele disponibilizou, respeitando os argumentos delas, uma explicação sintética das principais caraterísticas do conceito, usando para isso o gráfico já apresentado (Figura 5). 


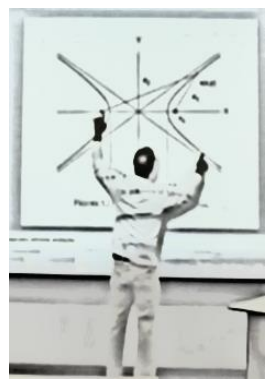

Quando eu pego um ponto qualquer na hipérbole e tenho esses dois pontos especiais chamados focos, a diferença da distância daqui [do ponto ao foco 1], com essa outra aqui [do ponto ao foco 2], é igual mesmo eu pegando outro ponto da hipérbole. Então essa diferença sempre é constante sem importar qual ponto eu pego. Essa é a caracterização do lugar geométrico chamado hipérbole. Agora o que temos que entender é como essa diferença se relaciona com o movimento da baleia e sua localização... (Áudio da fala do professor, na apresentação oral das alunas, ano).

Figura 5 - Professor explicando o significado matemático da hipérbole

Fonte: Vídeo da apresentação oral (2013)

O intuito é que, discutindo conceitualmente as caraterísticas matemáticas da hipérbole, o grupo conseguisse melhorar o processo de coordenação dos padrões de covariação do fenômeno com a correspondente correlação e, desse modo, fazer acontecer um novo aprendizado e o respectivo desenvolvimento cognitivo. Na explicação conceitual, o professor enfatiza que qualquer hipérbole deve ter a propriedade de que "todos os pontos $P$ que a ela pertencem, o valor absoluto da diferença de $P$ a dos pontos fixos chamados focos é uma constante" (Vídeo da apresentação oral, 2013). Tal "diferença constante" que define a hipérbole pode ser entendida como a "célula" ou, em palavras de Davidov (1988), o "abstrato concreto" que encapsula a forma geral e, por essa via, o conceito algébrico que permite estudar os problemas particulares sobre hipérbole. Desse modo, tanto o conceito algébrico, quanto a sua particularização mediante o problema sobre a localização de cetáceos em mar aberto é singularizado nas diferentes compreensões das alunas e do professor.

Pela experiência com o conceito, o professor sabe que nas equações e no gráfico apresentado pelas alunas deve estar codificada historicamente essa diferença constante e que deveria corresponder com a situação-problema particular ou fenômeno físico de variação. Isso explica sua insistência por fazer aparecer o conceito de diferença constante, como uma confluência dos campos de significação qualitativa do conceito como um geral e de, pelo menos, um particular. Ele entende que um passo importante na busca de sintonia das ações próprias da atividade de modelagem com as correspondentes ações das práticas legitimadas como algébricas é fazer com que as alunas ingressem no nível de compreensão do significado matemático da diferença constante, sem importar o registro de representação utilizado. Seguindo as ideias de Davidov (1988) isso indica uma ascensão do abstrato para o concreto.

Quando isso acontecer, supõe-se que há um ganho cognitivo das alunas, na medida em que lhes será possível operar analiticamente a forma geral e/ou estrutural da correlação entre quantidades indeterminadas por meio das técnicas de manipulação algébrica materializada na equação, passando a ter uma compreensão mais aprofundada da relação particular de 
covariação como caso particular. Uma forma de interpretar esse ganho cognitivo é considerar que as alunas ingressam em uma camada de significação de maior generalidade, abstração e concretude das práticas algébricas. Essa era uma das intenções do professor quando pediu para elas primeiro tentarem explicar qualitativamente a relação da hipérbole com o problema formulado e após disso as regras de operação quantitativa (“as passagens da equação”).

Fazer ingressar as alunas nessa área de prática algébrica usando contextos de projetos pedagógicos de modelagem não é um processo imediato nem transparente, daí a importância dos constantes atos instrutivos, para que os alunos consigam ser conscientes de que em meio à mudança (padrão de variação), há algo que permanece (invariante estrutural), condição necessária para ingressar à área de significação cada vez mais próxima de práticas algébricas. Por isso, para que os alunos percebam a relação geral, consigam operar com ela e compreendam seu papel no tratamento das várias particularidades que representa, devem participar ativamente em diversas atividades e com diferentes formas simbólicas, mesmo sem ter um domínio total de significação delas, pois, nas formas de interação social, é possível empregar mais símbolos do que realmente compreendemos. Esse exercício favorece o ingresso às diferentes camadas de significações esperadas.

$\mathrm{Na}$ ementa da disciplina não estava previsto trabalhar o conceito de hipérbole. Porém, a partir dessa situação emergente, para o professor foi necessário construir novos atos instrutivos, representados em outras atividades e situações-problema particulares para acrescentar, ao campo de significação das alunas, outras possibilidades de coordenação do padrão de variação "diferença constante" com a correspondente correlação codificada no conceito matemático de hipérbole.

\section{Considerações finais}

Nosso propósito neste trabalho visava discutir alguns aspectos relativos à constituição de práticas algébricas localizadas em contextos pedagógicos de produção de conhecimento. Quando falamos de prática algébrica, nos referimos a um conjunto particular de ações orientadas ao tratamento analítico de quantidades indeterminadas com o auxílio de diferentes artefatos culturais materiais e simbólicos. Para atingir o objetivo, no ano 2013 alunos de uma disciplina de Matemática para Biólogos da UNESP/Rio Claro/SP/Brasil, foram convidados a desenvolver Projetos Pedagógicos de Modelagem - PPM, convite que serviu de estímulo para fazer entrar em atividade a produção de conhecimento dos alunos. Pela natureza dessa perspectiva pedagógica, entendemos que nem sempre surge a necessidade e/ou a possibilidade 
de constituir práticas algébricas. Porém, de acordo com as caraterísticas específicas de cada projeto, podem ser criadas condições para que faça sentido constituí-las, principalmente pelo papel que têm na formulação e solução das situações-problema.

Argumentamos que, nesse contexto, as práticas algébricas se constituem como síntese de um processo de coordenação de padrões de variação entre as quantidades pertinentemente da situação-problema (um particular) com certas formas de correlação entre variáveis indeterminadas correspondentes (um geral), singularizada nas ações interpretativas e propositivas dos sujeitos envolvidos na atividade. Dado que tal coordenação não é um processo simples de realizar, o trabalho coletivo com a participação de atores com diferentes experiências na situação tratada, torna-se uma das principais estratégias para consegui-lo. Ressaltamos, em especial, aqueles atores que têm tido como objeto de reflexão e de pesquisa os aspectos relevantes da situação. Esse é um dos papéis que normalmente tem o professor enquanto acompanha os alunos no desenvolvimento dos PPM.

Uma das ações mais importantes destes atores mais experientes, em particular do professor, é propor diferentes tipos de ações mediante o uso dos artefatos disponíveis, principalmente simbólicos, orientando a surgir na consciência dos alunos (aprendizagem) as possibilidades de operar com os padrões de variação e correlação, relativas às situaçõesproblema formuladas. Propusemos que, na medida em que se consiga produzir, pelo menos, um padrão de correlação que relacione as variáveis indeterminadas associado ao padrão de variação definido, tem-se configurado uma forma geral que coloca à situação-problema em estudo como um caso particular e, por essa via, pode-se dizer que se constituíram ações com sentido e significado algébrico no contexto dos PPM. Isto é, práticas algébricas. Visto assim, podemos afirmar que as práticas algébricas constituídas nos PPM não estão totalmente submersas no campo de significação legitimado pela institucionalidade matemática como "algébrico", pois os sentidos e significados das formas de expressão da correlação/geral continuam impregnadas de sua significação variacional/particular.

Consideramos que essas dualidades não se apresentam de maneira excludente sob uma lógica de "ser" ou "não ser", mas dialética, como confluência de contrários que se negam e se desenvolvem mutuamente na singularidade das ações realizadas com o auxílio dos diferentes registros simbólicos com os que se produzem os diversos sentidos e significados. Uma possível interpretação para essa confluência de sentidos e significados que configuram as práticas algébricas no contexto dos PPM é em termos de zonas de trânsito semântico das ações dos atores, que podem estar mais ou menos próximos do caráter geral e fazem uso de registros simbólicos mais ou menos próximos de representações alfanuméricas (ver Figura 6). 


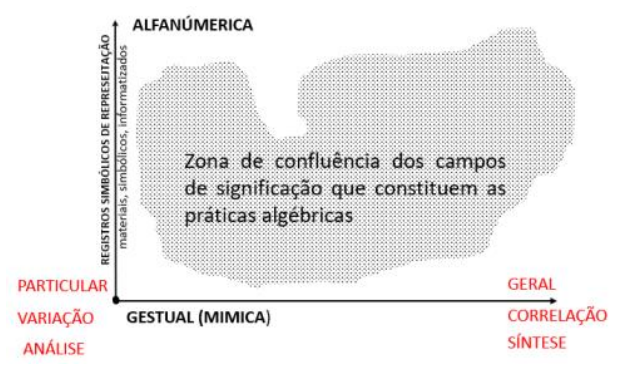

Figura 6 - Zona de constituição de práticas algébricas

Fonte: Os autores (2013)

Embora não fosse possível falar de todo o processo desenvolvido com as alunas na produção do PPM, com o episódio analisado pretendíamos ilustrar algumas formas de materialização, operação e transformação dos diferentes sentidos e significados das ações que constituem essa zona. Esboçamos assim, alguns dos movimentos nos espaços de significação pelos que foram transitando os atores humanos (professores, alunas e monitores), enquanto desenvolviam os PPM usando diferentes registros simbólicos de representação (atores não humanos).

De acordo com o exemplo discutido, nas representações simbólicas com que foi tratado o conceito de hipérbole, não apenas confluiu o padrão de variação (dinâmico) e a correspondente correlação (estático) da diferença constante entre quantidades, mas também a tensão de ser um argumento que dá solução ao problema particular sobre localização de cetáceos, com o fato de ser um invariante geral que pode ser usado para representar qualquer outra situação com caraterísticas semelhantes. Nessas condições, sugerimos que as práticas algébricas constituídas nos PPM podem ser assumidas com o papel de modelos matemáticos, pois como sugere Davidov (1988) e Kursanov (1966), um modelo deve comportar uma original "mistura" de evidências e abstrações, expressando relações intrínsecas gerais dos objetos modelados, mas garantindo a dedução e o tratamento das diversas formas particulares que os contextualizam.

\section{Agradecimentos}

Este artigo é produto da tese de doutorado em Educação Matemática desenvolvida pelo primeiro autor sob a orientação do segundo e que contou com o patrocínio da Asociación Universitaria Iberoamericana de Postgrado - AUIP - e o Programa de Ajuda ao Estudante do Estrangeiro - PAEDEX - da UNESP, Campus Rio Claro, SP, Brasil. Um agradecimento especial para estas entidades. 


\section{Referências}

ARAÚJO, J. Brazilian research on modelling in mathematics education. ZDM Mathematics Education, Karlsruhe, v. 42, n. 3-4, p. 337-348, 2010.

BEDNARZ, N.; KIERAN, C.; LEE, L. Approaches to algebra. Perspectives for research and teaching. Netherlands: Kluwer Academic Publish, 1996.

BLANTON, M.; KAPUT, J. Functional Thinking as a Route Into Algebra in the Elementary Grades. In: CAI, J.; KNUTH, E. (ed.). Early Algebraization: A Global Dialogue from Multiple Perspectives. New York: Springer, 2011. p. 5-21.

BORBA, M. Can modelling be taught and learnt? -A commentary. In: KAISER, G. et al. (Ed.). Trends in teaching and learning of mathematical modelling: ICTMA 14. New York: Springer, 2011. p. 3136.

BORBA, M. Coletivos Seres-humanos-com-mídias e a Produção de Matemática. In: I SIMPÓSIO BRASILEIRO DE PSICOLOGIA DA EDUCAÇÃO MATEMÁTICA, 2001, Curitiba. Anais... Curitiba: UFPR, PUCPR, UTP, 2001. p. 135-146.

BORBA, M.; VILLARREAL, M. Humans-with-Media and the Reorganization of Mathematical Thinking: information and communication technologies, modeling, visualization and experimentation. United State of America: Springer, 2005.

CAI, J.; KNUTH, E. Early Algebraization: a global dialogue from multiple perspectives. Berlin: Springer, 2011.

CANTORAL, R.; FARFÁN, R. M. Pensamiento y lenguaje variacional en la introducción al análisis. Epsilon: Revista de la Sociedad Andaluza de Educación Matemática “Thales", n. 42, p. 353-372, 1998.

CHARBONNEAU, L. From Euclid to Descartes: Algebra and its relation to geometry. In: BEDNARZ, N.; KIERAN, C.; LEE, L. (ed.). Approaches to algebra: perspectives for research and teaching. Netherlands: Kluwer Academic Publish, 1996. p. 15-37.

DANIELS, H. Vygotsky e a pedagogia. Tradução de Milton Camargo Mota. 1a ed. São Paulo: Ed. Edições Loyola, 2003.

DAVIDOV, V. La enseñanza escolar y su desarrollo psicológico. Moscu: Editorial progreso, 1988.

DUVAL, R. Semiosis y pensamiento humano: registros semióticos y aprendizajes intelectuales. Traducción de Myriam Vega Restrepo. 1a ed. Santiago de Cali: Editora Universidad del Valle, Instituto de Educación y Pedagogía, Grupo de Educación Matemática, 1999.

ENGESTRÖM, Y. Activity theory and individual and social transformation. In: ENGESTRÖM, Y.; MIETTINEN, R.; PUNAMAKI, R.L. (ed.). Perspectives on activity theory. United States of America: Cambridge Unversity Press, 1999. p. 19-38.

GODINO, J. et al. Naturaleza del Razonamiento Algebraico Elemental. Bolema, Rio Claro, v. 26, n. 42B, p. 483-511, 2012.

KAISER, G.; SRIRAMAN, B. A global survey of international perspectives on modelling in mathematics education. ZDM Mathematics Education, Karlsruhe, v. 38, n. 3, p. 302-310, 2006. 
KIERAN, C. Research on the learning and teaching of algebra: A Broadening of Sources of Meaning. In: GUTIÉRREZ, A.; BOERO, P. (ed.). Handbook of Research on the Psychology of Mathematics Education Past, Present and Future. UK: Sense Publishers, 2006. p. 11-50.

KIERAN, C. The core of algebra: reflections on its main activities. In: STACEY, K.; CHICK, H.; KENDAL, M. (ed.). The future of the teaching and learning of algebra: the 12th ICMI study. New ICMI Study Series. United Estate of America: Kluwer Academic Publishers, 2004. v. 8. p. 21-33.

KLEIN, J. Greek mathematical thought and the origin of algebra. Tradução Eva Brann. United Estate of America: Dover publications, 1992.

KURSANOV, G. Materialismo dialéctico y el concepto. México D.F.: Editorial Grijalbo, 1966.

LEONTIEV, A. N. Actividad, conciencia y personalidad. Buenos Aires: ediciones ciencias del hombre, 1978.

LINS, R. A framework for understanding what algebraic thinking is. 1992. Tese (Doutorado em filosofia) - University of Nottingham, 1992. Disponível em: <http://etheses.nottingham.ac.uk/3227/1/316414.pdf>. Acesso em: 05 jul. 2012.

LINS, R.; GIMENEZ, J. Perspectivas em aritmética e álgebra para o século XXI. 4. ed. Campinas: Papirus Editora, 2001.

LINS, R.; KAPUT, J. The Early Development of Algebraic Reasoning: The Current State of the Field. In: STACEY, K.; CHICK, H.; KENDAL, M. (ed.). The Future of the Teaching and Learning of Algebra The 12th ICMI Study. United Estate of America: Kluwer Academic Publishers, 2004. p. 4770 .

MALHEIROS, A. P. A produção matemática dos alunos em um ambiente de modelagem. 2004. Dissertação (Mestrado em educação matemática) - Universidade Estadual Paulista, Rio Claro, 2004. Disponível em: <http://www.rc.unesp.br/gpimem/downloads/dissertacoes/malheiros_aps_me _rcla.pdf $>$. Acesso em: 25 jun. 2012

MALHEIROS, A. P. Modelagem Matemática e Pedagogia de Projetos: possíveis interseções. In: ENCONTRO NACIONAL DE EDUCAÇÃO MATEMÁTICA, 9., 2007, Belo Horizonte. Anais... Belo Horizonte: UFMG, 2007. p. 1-15.

MEDWIN, H.; CLAY, C. Fundamentals of Acoustical Oceanography. USA: Academic Press, 1998.

MUNZÓN, N.; BOSCH, M.; GASCÓN, J. El problema didáctico del algebra elemental: un análisis macro-ecológico desde la teoría antropológica de lo didáctico. v. 4, n. 2, 2015. Disponível em: <http://dx.doi.org/10.17583/redimat.2015.1386>. Acesso em: 04 jun. 2016.

OBANDO, G.; ARBOLEDA, L. C.; VASCO, C. E. Filosofía, matemáticas y educación: una perspectiva histórico - cultural en educación matemática. Ciencia, Ingeniería y Educación. Bogotá, v. 3, n. 20, p. 73-90, 2014.

PONTE, J. P. DA; GUIMARÃES, H. M. Notes for a History of the Teaching of Algebra. In: KARP, A.; SCHUBRING, G. (Ed.). Handbook on the History of Mathematics Education. New York: Springer, 2014. p. 459-472.

POSADA, F. et al. (Org.). Pensamiento variacional y razonamiento algebraico. 1. ed. Medellín, Colombia: Secretaría de Educación para la Cultura de Antioquia, 2006.

POSADA, F.; VILLA, J. Propuesta didáctica de aproximación al concepto de función lineal desde una perspectiva variacional. 2006. Tésis (Maestría en educación) - Universidad de Antioquia, 
Medellín, 2006. Disponível em: <http://tesis.udea.edu.co/bitstre am/10495/7093/1/FabianPosada_2006_didacticafuncionlineal.pdf >. Acesso em: 25 jun. 2010.

PRADO JUNIOR, C. Dialética do conhecimento. 6. ed. São Paulo, Brasil: Editora Brasiliense, 1980.

RADFORD, L. Algebraic thinking from a cultural semiotic perspective. Research in Mathematics Education, British Society for Research into Learning Mathematics, v. 12, n. 1, p. 1-19, 2010a. Disponível em: <http://www.luisradford.ca/pub/22_RME2010Algebraicthi nkingfromaculturalsemioticperspective.pdf>. Acesso em: 12 mai. 2015

RADFORD, L. Layers of generality and types of generalization in pattern activities. 2010b. Disponível: <http://www.luisradford.ca/pub/23_PNA2010Layersgenerality.pdf>. Acesso em: 12 mai. 2015

RADFORD, L.; SABENA, C. The Question of Method in a Vygotskian Semiotic Approach. In: BIKNER-AHSBAHS, A.; KNIPPING, C.; PRESMEG, N. (Ed.). Approaches to Qualitative Research in Mathematics Education: examples of methodology and methods. Springer, 2015. p. 157-182.

RIBEIRO, A.; CURY, H. Álgebra para a formação do professor: explorando os conceitos de equação e de função. 1. ed. Belo Horizonte, Brasil: Autêntica Editora, 2015.

STACEY, K.; CHICK, H.; KENDAL, M. The future of the learning and teaching of algebra: the 12th ICMI study. Boston: Kluwer Academic Publishers, 2004.

STREECK, J.; MEHUS, S. Microethnography: the study of pratices. In: FITCH K.; SANDERS R. Handbook of language and social interaction. Mahwah, New Jersey: Lawrence Erlbaum, 2005. p. 381-404.

SUTHERLAND, R.; ROJANO, T.; LINS, R. Perspectives on School Algebra. New York: Kluwer Academic Publishers, 2002.

VÁZQUEZ, A. Filosofia da práxis. Tradução Maria Encarnación Moya. Edição em português Sergio Dante Julião da Silva. São Paulo: CLACSO Livros, 2007. (Pensamento social latino-americano).

VIÈTE, F. The analytic art: nine studies in algebra, geometry and trigonometry from tue Opus restitutae mathematicae analyseos, seu algebrâ Novâ. Tradução Richard Witmer. Ohio: The Kent state University Press, 1983.

VYGOTSKY, L. The instrumental method in psychology. In: WERTSCH, J. (Org.). The concept of activity in Soviet psychology. Sharpe ed. Armonk, N. Y.: [s.n.], 1981. p. 134-143.

VYGOTSKY, L. Thought and language. Tradução Alex Kozulin. USA: The Massachusetts Institute of Technology, 1986. 